\title{
Kernos
}

Revue internationale et pluridisciplinaire de religion grecque antique

12| 1999

Varia

\section{Cadavres exquis}

\section{Pierre Somville}

Édition électronique
URL : http://journals.openedition.org/kernos/710

DOI : $10.4000 /$ kernos.710

ISSN : 2034-7871

\section{Éditeur}

Centre international d'étude de la religion grecque antique

\section{Édition imprimée}

Date de publication : 1 janvier 1999

Pagination : 73-83

ISSN : 0776-3824

Référence électronique

Pierre Somville, "Cadavres exquis », Kernos [En ligne], 12| 1999, mis en ligne le 13 avril 2011, consulté le 21 avril 2019. URL : http://journals.openedition.org/kernos/710; DOI : 10.4000/kernos.710 
Kernos, 12 (1999), p. 73-83.

\title{
Cadavres exquis*
}

\begin{abstract}
Cypris, pour exciter de vifs regrets dans l'âme du vainqueur, avait conservé à Pentbésilée, même après son trépas, tous les charmes qui l'avaient fait admirer pendant sa vie.
\end{abstract}

(Quintus de Smyrne)

On meurt beaucoup dans l'Iliade. Au point que le « divin » Scamandre en vient à se plaindre de ce que ses eaux soient saturées de corps morts et son cours presque interrompu. Seul l'incendie, suscité par Héphä̈stos, parviendra à mettre fin, pour un temps, aux excès meutriers d'Achille. Mais ce n'est là qu'un épisode (Chant XXI). En fait, c'est après chaque affrontement que les cadavres couvrent la plaine de Troie, de part et d'autre des lignes. Or, pour la masse anonyme de ceux qui sont tombés, Homère ne mentionne, en passant, que des funérailles expéditives. On lave et on brûle, vite, les corps qui jonchent la lice comme flambaient déjà les bûchers de l'épidémie au début du chant I. Pas d'epithaphios, donc, pour cette piétaille qui n'est pas encore la reine des batailles, rien qui annonce ou préfigure les célébrations rituelles, et annuelles, qu'instaurera la cité démocratique - et dont Nicole Loraux a, par ailleurs, si bien parlél. Outre le fait que l'incinération soit donc de règle pour Homère, ces brefs passages ne nous apprennent pas grand-chose sur la mort des guerriers ou sur ses suites immédiates.

Toutefois, certains héros ont droit à un traitement de faveur, digne sans doute de leur bravoure. Je pense évidemment à Sarpédon, à Patrocle et à Hector. On se souvient des conditions particulièrement dramatiques de leur mort : Sarpédon, le Lycien, aimé de Zeus mais sacrifié quand même, tombe sous les coups de Patrocle, lequel ne tarde pas à subir le même sort, victime de son bybris, qui lui a fait pousser l'affrontement bien au-delà de l'efficacité requise. Et il succombe devant un Hector impitoyable. Achille, enfin, trouvant comme d'instinct le défaut de la cuirasse, à l'angle de l'encolure et de la clavicule, fait mordre la poussière au Prince de Troie. Toujours après des dis-

* En marge de cette relecture solaire de l'tliade, je me dois de remercier Mme Marie-Hélène Marganne, à qui rien de la médecine antique n'est étranger, pour les précieuses références qu'elle a bien voulu me communiquer.

1 L'invention d'Athènes, Paris, 1981. Voir aussi, à propos d'incinération et de crémation, J. Deмот, La crémation et le séjour des morts chez les Grecs, Bruxelles, 1909 (reprise d'un texte paru dans les Mémoires de la Société d'antbropologie de Bmixelles, 27, 6, 1908). 
cours alternés où l'insulte, la prophétie et la menace se le disputent de part et d'autre. Comme si la parole, non moins que le marbre ou que l'airain, que le fer ou que le bronze, participait de l'agôn et de son mémorial. De l'invective au coup de lance et au coup de glaive, jusqu"au coup de burin dans la stèle et jusqu'au verbe du poète, les mots volent comme des traits, et restent, contrairement à ce que dit le proverbe, non d'abord parce qu'ils sont écrits, mais parce qu'ils auront été proférés, récités, répercutés, redits et retenus, d'abord, de mémoire.

Mais tout ceci est bien connu des lecteurs de l'Iliade, surtout s'ils ont un peu pratiqué, de surcroît, Severyns, Vernant, Detienne, Svenbro, Bader, Wathelet... Ce que j'aimerais dire, après l'avoir examiné, c'est combien la mort exemplaire de ces trois héros est suivie, non tant de funérailles extraordinaires, comme c'est surtout le cas pour Patrocle qui en occupe tout le Chant $\mathrm{XXIII}^{2}$, mais d'un traitement très particulier des corps, précédant toute forme canonique de rites funéraires. Ce point me semble avoir été souvent négligé par les commentateurs et les archéologues, plus soucieux d'exactitudes littérales ou matérielles que de projections de l'imaginaire sur le réel, ou sur certaines réalités.

Mais procédons par ordre et commençons par les textes. Et d'abord Sarpédon : touché au péricarde et une fois abattu, c'est Patrocle, un pied sur sa victime, qui retire son trait de la blessure et ramène à la pointe de sa lance, comme le dit le Poète, à la fois le péricarde et l'âme du héros mort. Zeus a laissé faire.

Autour du corps, la mêlée qui s'ensuit est des plus confuses et des plus brutales: Glaucos et les deux Ajax notamment s'y affrontent. Zeus laisse encore faire, comme pour donner du relief à la mort de son fils. Le combat pour les armes du héros mort tourne finalement à l'avantage des Grecs. Patrocle pratique alors ce premier outrage qui consiste à dépouiller le mort. Alors, Zeus intervient. Il ordonne à Apollon, un autre de ses fils, d'éviter au corps mort de pires outrages tels que le dépeçage du vaincu (maschalismos) ou simplement la putréfaction aidée par les mouches et les vers en attendant que les traditionnels charognards «les oiseaux et les chiens » fassent leur office. Au contraire, ce que doit faire Apollon, c'est d'abord éponger le corps du sang qui le macule, ensuite en enlever les traits nombreux qui sont venus s'y ficher lors des différents combats, ensuite encore le laver et, enfin, le couvrir d'ambroisie. Ainsi Mort et Sommeil, les deux jumeaux, pourront le

2. Voir l'étude d'A. Schnapp-Gourbeillon, Les funérailles de Patrocle, in G. Gnoli et J.-P. VERnant (éds), La mont, les morts dans les sociétés anciennes, Cambridge, 1982, p. $77-$ 88. On y découvre l'aspect extraordinaire - et excessif en tous sens - de ces funérailles peu communes. Voir aussi, bien sûr, E. Rohde, Psycbe, Paris, 1928, trad. A. Reymond, p. 15-23. Et notamment p. 23 : "Homère ne connaît d'autre mode de sépulture que par le feu ». A. Severyns, pour sa part, dans Grèce et Procbe-Orient avant Homère, Bruxelles, 1960 , p. 186-188, se réfère au rituel funéraire des Hittites à Boghazköy, présentant quelque analogie avec les funérailles de Patrocle. 
transporter chez lui en Lycie où l'enseveliront les siens, signalant sa mémoire par une tombe et par une stèle. Voici le texte :

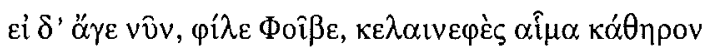

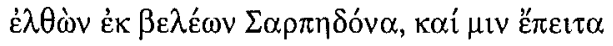

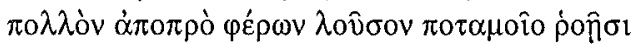

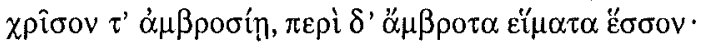

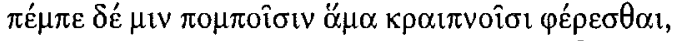

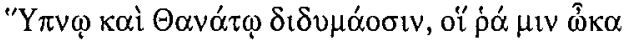

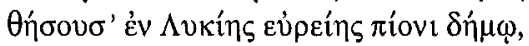

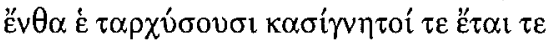

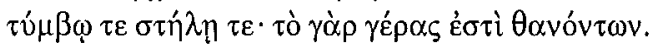

(XVI, 667-675 [éd. Monro-Allen, Oxford, 1902])

Va maintenant, cher Phœbos, va soustraire aux traits Sarpédon, efface sur lui le sang noir; puis porte-le bien loin, et lave-le dans l'eau courante d'un fleuve. Oins-le ensuite d'ambroisie, revêts-le de vêtements divins : enfin remets-le aux porteurs rapides qui doivent l'emporter, Sommeil et Trépas, dieux jumeaux. Ils auront tôt fait de le déposer au gras pays de la vaste Lycie, où ses frères et parents l'enterreront dans un tombeau, sous une stèle, puisque tel est l'hommage dû aux morts (trad. P. Mazon).

Nous remarquerons d'abord que l'intervention d'Apollon n'est sans doute pas amenée ici tant par son amitié proverbiale en faveur des Troyens et de leurs alliés que par sa fonction de dieu guérisseur. L'archer qui blesse ou qui tue est aussi celui qui sait soigner et même, parfois, prodiguer aux morts les soins qui leur conviennent, Laver le sang caillé et baigner le corps, bien loin dans un fleuve, sont sans doute les premiers traitements réservés aux défunts. Ce sont aussi les plus banals, comme d'enlever du corps les traits et flèches qui le hérissent. Plus surprenante est cette « onction d'ambroisie » prescrite par Zeus, afin d'éviter au corps les outrages naturels évoqués plus haut. Il s'agit, en quelque sorte, de lui donner couleur d'immortalité, puisque l'ambroisie est cette substance sacrée qui assure aux dieux leur pérennité, principale différence d'avec les hommes " mortels ».

Ajoutons que ces funérailles lyciennes sont rendues possibles grâce au transport, assurément exceptionnel, des deux dieux psychopompes dont la présence même atteste que si le sommeil ressemble à la mort, parfois à s'y méprendre - la mort pourrait bien n'être aussi qu'une forme de sommeil pour cet « endormi » en voie d'héroïsation (c'est-à-dire de « semi-divinisation ») qu'est ici le héros couché. La filiation divine de Sarpédon trouverait là comme une forme de légitimation. Quant au rituel proprement dit, il est désigné par le verbe $\tau \alpha \rho \chi v ́ \omega$, d'emploi assez rare, que je préférerais paraphraser par ensevelissement plutôt que par inhumation, ou même enterrement, comme le fait Mazon. Rien ne dit en effet que le corps doive être inhumé : la tombe et la stèle peuvent accompagner n'importe quel type de rituel. Mais, en tout cas, et ceci est explicite, - il ne sera pas brûlé. 
Passons à Patrocle. Il est midi et le combat fait rage (XVI, 777 sq.). Mais quand s'amorce le couchant, la chance tourne; le héros solaire voit se sceller son destin. Et c'est le dieu, solaire lui-même, Apollon, qui vient, d'un coup du plat de la main dans le milieu du dos, mettre un terme à la course trop brève de son avatar, tristement mortel. Il s'est enveloppé d'une nuée paradoxale qui le dissimule et lui permet de faire perdre la tête à sa victime. Quant au coup de lance, c'est le guerrier Euphorbe qui le lui assène, par derrière lui aussi, avant qu'Hector l'achève d'un coup de pique au ventre. Incapable de résister aux tentations de l'bybris, vraie préfiguration de l'bamartia tragique, son vainqueur l'insulte alors en lui promettant d'être, sur place, dévoré par les

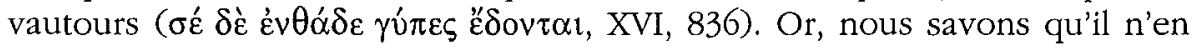
sera rien.

Les premiers soins prodigués au corps de Patrocle, après les premiers cris et larmes d'Achille au début du chant XVIII consisteront en pratiques de lavage, onction d'huile et traitement de plaies par un onguent « de fraîcheur » ('́vvéwpos) $)^{3}$. Ce sont les servantes d'Achille qui s'acquittent de cette toilette funèbre, que je serais tenté d'appeler le cérémonial profane, par opposition à l'intervention - sacrée - de Thétis, au début du chant XIX, qu'il nous faut à présent considérer.

Sortant de la mer, comme chaque fois qu'elle doit venir consoler son fils, son « grand enfant », Thétis, la mère, marine en l'occurrence, lui apporte ses nouvelles armes, et notamment le fameux bouclier aux cinq épaisseurs, dûment façonnés par Héphaïstos à la fin du chant précédent. Elle lui promet surtout de protéger le corps de son ami. Quand Achille s'inquiète des ravages que les mouches et leurs larves pourraient exercer en s'introduisant dans les plaies du défunt, elle lui promet un traitement apómuios ${ }^{4}$ qui le protégera, quand bien même le corps « devrait gésir sur la grève une année durant » lui dit-elle (XIX, 24-27; 30-33).

Vient enfin le traitement, précis, net, énoncé en deux vers d'une sobriété toute chirurgicale :

Quant à Patrocle, c'est l'ambroisie et le rouge nectar

Que goutte à goutte elle lui met dans les narines

Pour que la chair lui reste intacte

$(\mathrm{XIX}, 38-39)$

La mère qui donne la vie et, bien que divine, donne également la mort, peut donc aussi dispenser cette forme d'immortalité mineure, post mortem, qui rend inaltérable, fût-ce pour une année, la dépouille bien-aimée. Or, elle

3 Je choisis ce sens, contrairement à Mazon qui y voit un onguent « de neuf ans » (tout en mentionnant en note l'autre hypothèse).

4 Telle est aussi l'une des multiples épiclèses «fonctionnelles" de Zeus. Voir H. UsEner, Götternamen. Versucb einer Lebre von der religiösen Begriffsbildung, Bonn, 1896, p. 260. 
est déesse et c'est à l'ambroisie qu'elle recourt comme Apollon pour le corps de Sarpédon, y adjoignant le nectar, introduit, lui aussi, au fond des fosses nasales du défunt.

Hector, enfin. Pour ce protégé de Zeus et d'Apollon, l'épreuve idéenne de la psychostasie ante mortem est néanmoins funeste. L'inexorable doit s'accomplir. Le père des dieux lui-même n'y peut rien. La lance d'Achille lui perce le haut du torse, juste à côté de la trachée de sorte qu'il puisse encore supplier son vainqueur, in articulo mortis : « qu'il ne laisse pas les chiens le dévorer... » (XXII, 339). Mais c'est lui qu'Achille impitoyable traite de chien (345) avant d'adresser à son corps déjà mort cette parole, comme un couperet :

- Meurs! Té $\theta v \alpha \theta_{\mathrm{r}}$

Or, malgré la promesse (ou plutôt la menace) proférée par Achille « nul n'écartera les chiens de sa tête » (348), malgré les craintes d'Andromaque éplorée " les vers grouillants après les chiens repus vont dévorer ton corps » (509), une double intervention divine vient protéger le corps, outragé comme on sait, les chevilles percées, traîné dans la poussière, derrière le char (395404).

Et c'est au chant XXIII, en parallèle aux apprêts funèbres consacrés à Patrocle et contrairement à une nouvelle menace de "dévoration " par les chiens proférée par Achille (182-183) que l'aède, apparemment pris de pitié, récite les vers que voici :

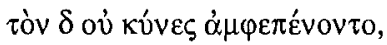

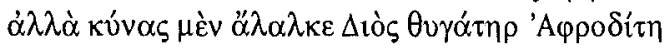

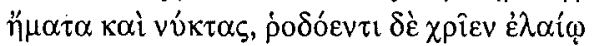

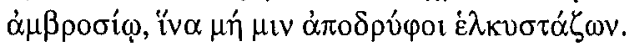

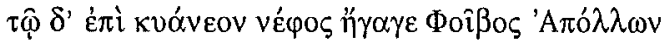

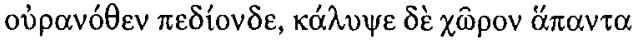

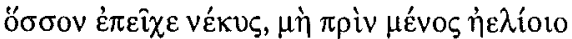

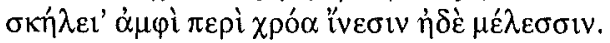

(XXIII, 184-191)

Autour d'Hector cependant les chiens ne s'affairent pas. La fille de Zeus, Aphrodite, nuit et jour, de lui les écarte. Elle l'oint d'une huile divine, fleurant la rose, de peur qu'Achille lui arrache la peau en le traînant. Pour lui, Phœbos Apollon amène du ciel sur la plaine une nuée sombre et dérobe aux yeux tout l'espace qu'occupe le corps : il ne veut pas que l'ardeur du soleil lui déssèche trop vite la peau autour des tendons et des membres (trad. P. Mazon).

Arrêtons-nous un instant. Aphrodite, d'abord, « de jour et de nuit » écarte du corps les chiens errants, comme une paradoxale (sinon inversive) gardienne du monde des morts. Il est vrai que l'amour et la mort, comme plus haut la mort et le sommeil, vivent de secrètes accointances. La coincidentia 
oppositorum n'a pas attendu Nicolas de Cuse, son meilleur théoricien, pour signifier les relations des dieux et des hommes comme de l'espace profane affronté à l'abaton du sacré. Aphrodite, donc, comme une sphinge postée à l'orée d'un au-delà des âmes, garde aussi le corps mort et le préserve. Elle y pratique même le traitement générique que nous commençons à connaître :

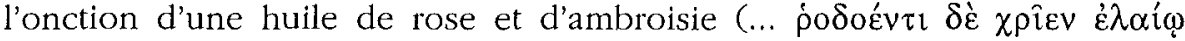
$\dot{\alpha} \mu \beta \rho o \sigma i ́(\omega)$ dont le but avoué est de protéger la peau et d'éviter que le corps ne soit écorché. Survient alors Apollon, dans le rôle non moins paradoxal de

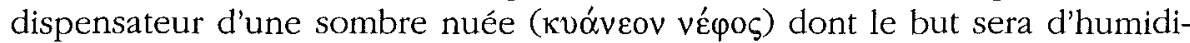
fier le cadavre et d'assurer à la peau toute sa souplesse. Ainsi le dieu solaire devient son propre antagoniste. Il s'offusque et répand la nuée plombée, gris sombre comme les proues, le ciel et la mer, juste avant l'orage. Il est vrai qu'au chant I, dans son grand courroux contre les Grecs et lorsqu'il faisait chanter sinistrement en son carquois les flèches, il était dit déjà « semblable à la nuit ».

Ce passage nous rappelle qu'Apollon, comme le soleil qui donne la vie, peut aussi donner la mort ou la parfaire en desséchant les cadavres, les privant de cette humidité vitale qui assure l'irrigation des tissus. Mais le dieusoleil qui tue peut également se transformer, pour la cause, en son propre contraire : une pulvérisation d'eau sombre qui donne ou qui préserve la vie, en son apparence à tout le moins 5 .

Ce double paradoxe du rôle proprement funèbre confié à Aphrodite et Apollon, les divinités les plus liées, - surtout chez Homère, - à l'or et à la lumière, nous met en présence d'un coefficient d'inquiétante étrangeté qui se devait d'être souligné. Les ambiguités du sacré se mesurent à ce prix.

Quant à l'efficacité du traitement, Hermès, grand spécialiste des passages, nous en apporte le témoignage lorsque, pour rassurer Priam, il lui dit qu'après onze jours d'exposition sauvage et d'outrage, le corps de son fils est resté intact. Ecoutons-le :

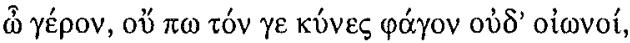

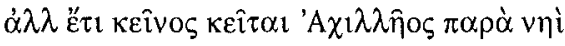
$\alpha$

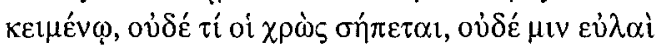

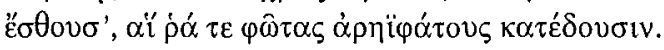

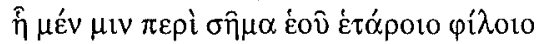

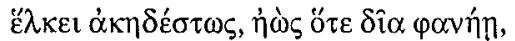

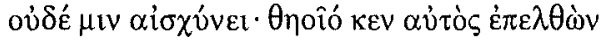

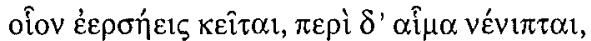

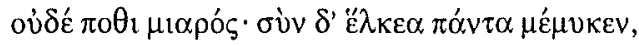

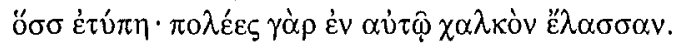

La remarque n'a pas échappé au scholiaste du Venetus B qui invoque, pour éviter

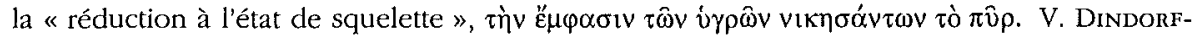
MaAss, Oxford, 1888, ad locum, VI, p. 114 (=Erbse, Berlin, 1977, V, pp. 399-400). 


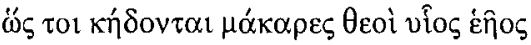

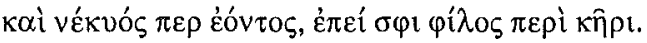

(XXIV, 411-423)

Non, vieillard, les chiens ni les oiseaux ne l'ont pas dévoré; il est toujours près de la nef d'Achille, tel quel, dans sa baraque. Voici la douzième aurore qu'il est là, étendu à terre, et sa chair ne se corrompt pas; ni les vers ne l'attaquent, ces vers qui dévorent les mortels tués au combat. Sans doute, Achille, chaque jour, le traîne brutalement tout autour de la tombe de son ami, à l'heure où paraît l'aube divine : il ne l'abîme pas pour cela. Tu l'approcherais, tu verrais toi-même comme il est là, tout frais, le sang qui le couvrait lavé, sans aucune souillure, toutes ses blessures fermées, toutes celles qu'il a reçues - et combien de guerriers ont poussé leur bronze sur lui ! C'est ainsi que les dieux bienheureux veillent sur ton fils, même mort. Il faut qu'il soit cher à leur cour (trad. P. Mazon).

Pour terminer, trois notations encore. Un retour d'Apollon, d'abord, plus attendu et qui protège le corps d'Hector en le couvrant " de son égide d'or " (XXIV, 18-21); le fait que ce que j'ai appelé'le cérémonial profane dans le cas de Patrocle suit ici l'intervention divine, au lieu de la précéder, lorsqu'Achille ordonne à ses servantes de laver et de frotter d'huile le corps avant qu'il soit remis à Priam, dûment couvert d'une tunique et d'une pièce de lin (587-588). Enfin, Hécube en digne mère de Cassandre, ne manque pas de célébrer la fraîcheur du corps, comme couvert de rosée ('́poń $\epsilon$ ss), qui vient d'être remis aux siens, « gisant tout semblable à celui que le dieu à l'arc d'argent, Apollon, vient d'abattre de ses douces flèches » (758-759).

Il y a là, me semble-t-il, un bel effet de batterie, non seulement rhétorique, où l'euphémisme, la litote et l'oxymore s'allient à la voyance, typiquement apollinienne, du dieu torve qui tue en douceur, qui extermine et qui préserve, qui fait peur et qui attire.

Après ce bref rappel, quelques considérations thanatologiques. Je dirai d'emblée que je n'ai pas l'intention d'énumérer ou de classer plus avant les cadavres, ainsi que l'a fait récemment Bernard Deforge pour les Tragiques ${ }^{6}$. Je ne m'aventurerai pas davantage dans les méandres archéologiques des rites funéraires en divers lieux de l'âge du bronze ou de l'âge du fer ${ }^{7}$. Ce qui m'importe est l'effet de filigrane que l'on peut lire sous la mention de ces interventions divines que nous avons vues et dont le but était de préserver une apparence de vie aux corps morts des trois héros. Ce sont évidemment trois cas d'élection. Le fait que des divinités, dieux ou déesses, en soient les auteurs suffit d'ailleurs à en marquer le caractère d'exception.

6 Le Festival des cadaures, Paris, 1997 (Coll. Vérité des mythes).

7 Voir notamment D. Kurtz et J. BoArdman, Greek burial customs, New York, 1971. 
La bonne littérature sur la question nous apprend généralement que les techniques de momification, et même d'embaumement, étaient inconnues des Mycéniens, et au-delà, jusqu'à l'époque géométrique, voire orientalisante $^{8}$. Il est vrai qu'on n'en trouve pas (ou peu ?) de traces matérielles ou physiques au niveau des objets que manipulent les archéologues, paléontologues, anthropologues et autres paléo-ostéologues?.

C'est ainsi, par exemple, qu'arguant du climat et, implicitement, du silence (quasi) total des sources archéologiques, Antje Krug, dans un ouvrage récent, écrit sans sourciller que, contrairement aux civilisations de l'Amérique pré-colombienne ou de l'Égypte, « les cultures classiques étaient étrangères à de telles formes de conservation ${ }^{10}$. Dans le même sens, Donna Kurtz et John Boardman concluaient déjà à la même absence, tant en Grèce d'Asie que sur le Continent, mentionnant seulement la conservation, exceptionnelle à vrai dire (et pour des raisons de transport lointain), de deux rois de Sparte, l'un dans le miel, l'autre dans la cire $^{11}$. Les mêmes auteurs signalent cependant, - après avoir rappelé qu'à leur avis les pratiques homériques de lavage et d'onction des corps avaient « une valeur plus pratique que rituelle »-que le Poète « a pu entendre parler d'embaumement, ou à tout le moins de mesures de préservation temporaire d'un corps » (p. 186). Jean-Pierre Vernant, pour sa part, dans son étude sur « La belle mort et le cadavre outragé dans l'Iliade » mentionne seulement in fine que « les dieux se servent, dans les gestes de lavage et d'embellissement que pratiquent les hommes, d'onguents divins $\gg . . .12$. Peut-être est-ce là restreindre la spécificité de l'action imputée aux dieux, malgré l'allusion aux « onguents », et la banaliser en quelque sorte en la ramenant à la «pratique des hommes ». Ce n'est sans doute pas là ce que voulait nous faire entendre le Poète.

Par ailleurs, qu'il ait pu entendre parler d'embaumement et de mesures de préservation ( ... may have known of ») nous semble trop peu dire devant la précision des termes employés et la mise en valeur des situations évoquées. De plus, la réduction toute pragmatique imputée encore par Kurtz et Boardman aux pratiques de traitement des corps, juste après la mort, semble relever d'un esprit positiviste, hélas courant en la matière. Au reste, si j'ai appelé « profanes » ces actions, c'était au regard des interventions divines qui les suivent, car il demeure acquis que tout traitement post mortem participe déjà du rite, ce clivage qui, depuis le paléolithique, fonde l'anthropologie en faisant du corps mort un objet de vénération plutôt que de le destiner à être mangé, consommé ou simplement jeté aux ordures.

\footnotetext{
8 Kurtz - BoARDMan, op. cit. (n. 7), p. 31 sq.

9 Voir R. ARnotr, Disease, Healing and Medicine in the Aegean Bronze Age, Leiden, Brill, 1998.

10 A. Krug, Heilkunst und Heilkult. Medizin in der Antike, Munich, Beck, 19932 , p. 16.

11 Op. cit. (n. 7), p. 191.

12 Voir La mort, les morts ..., op. cit. (n. 1), p. $45-76$ (spéc. p. 71).
} 
Parlons à présent de l'ambroisie, et du nectar. La substance d'immortalité $(\dot{\alpha} \mu \beta \rho \circ \sigma i \alpha)$ est mentionnée dans les trois cas qui nous occupent. Le nectar, une fois seulement, est ajouté à l'ambroisie lors de l'intervention de Thétis sur le corps de Patrocle. Or, si l'aura sémantique du premier de ces termes est particulièrement lumineuse, grâce à une étymologie certaine, $\dot{\alpha}-\mu \beta \rho$ orí $\alpha$ évoquant d'emblée l'« im-mortalité », celle du nectar l'est beaucoup moins. La boisson traditionnelle des dieux leur assure sans doute aussi l'immortalité, mais la chose ne se lit pas immédiatement par transparence. Loin de là. Les principales accointances linguistiques du terme grec avec le sanscrit, le tokharien ou le hittite semblent connoter les dieux et la mort, peut-être aussi l'absence de mort, de même que le trafic ou le transport des morts. Ceci n'est pas sans intérêt, mais aucun argument décisif ne semble s'imposer, au regard de la grammaire comparée en tout cas. La réédition du Bailly, en 1950, notait déjà « terme religieux sans étymologie claire ». Un quart de siècle plus tard, dans la notice de son Dictionnaire étymologique de la langue grecque, P. Chantraine concluait : «pas d'étymologie établie ${ }^{13}$.

Une hypothèse récente pourrait cependant venir nous éclairer. L'auteur en est R. Drew Griffith et voici ce qu'il nous propose : un mot égyptien de radical - ntr - désigne à la fois la divinité et un sel que l'on peut identifier au

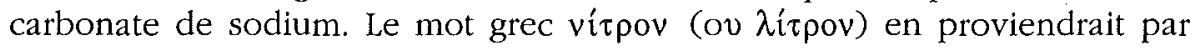
emprunt direct. D'autre part, le même radical, adopté par le phénicien avec renforcement consonantique en - $\mathrm{tk}$ - donne un radical - netkr - dont le mot grec qui nous occupe serait le dérivé, moyennant une métathèse consonan-

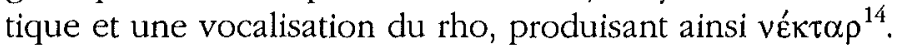

La démonstration linguistique est astucieuse et convaincante, et par effet de parallélisme elle nous évoque le cas de ces doublets savants directement empruntés au latin de la renaissance, à côté de termes issus du même mot latin mais qui ont été soumis aux métamorphoses (plus ou moins lourdes) de la phonétique évolutive. Ainsi captif/chétif; roture/rupture; purgation/purge, etc. On ne s'étonnera donc pas de la double voie suivie par l'évolution du « ntry » égyptien. Quant à la sémantique, comme nous l'ont si bien appris nos maîtres Fohalle et Knobloch, après Michel Bréal, elle est aussi indissociable de la grammaire comparée que les réalités désignées par les mots, pistés dans leurs voies évolutives souvent imprévisibles. Ainsi le « sel d'immortalité » ( $\mathrm{Na}_{2}$ $\mathrm{CO}_{3}$ ) est un ingrédient constitutif bien connu, notamment depuis Hérodote, dans le processus de momification en Égypte ${ }^{15}$. Que la notion de divinité ou de divinisation y soit première et celle du nitre ou natron immédiatement inférée par effet de métonymie, est une chose possible et même probable. En certaines matières, le « sens premier » ne se doit pas d'être toujours « maté-

13 III, Paris, 1974, p. 741-742. Voir dans le même sens H. FrIsk, Griecbisches etymologiscbes Wörterbuch, II (lieferung 14), Heidelberg, 1963, p. 300-301 et E. BorsacQ, Dictionnaire étymologique de la langue grecque, Paris, 1916, p. 660-661.

14 R. Drew GrifFith, Nektar and Nitron, in Glotta, 72 (1994), p. 20-23.

15 HDT., II, 86, 20-21. 
riel ». En tout cas, notre nectar homérique semble trouver ici à la fois une étymologie plausible et une identification substantielle, dont nous ne disposons toujours pas pour l'ambroisie, au sens pourtant si évident ${ }^{16}$.

Rappelons encore deux choses. La première, connue par Hérodote, nous apprend que dans la chirurgie mortuaire des Égyptiens la décérébration se faisait par le nez ${ }^{17}$. Or, on se souvient que c'est au fond des narines que Thétis instille " ambroisie et rouge nectar » à Patrocle. En second lieu, la déesse s'offre à garantir l'intégrité du corps pendant un an. Si pareille promesse ne relève pas de l'« exagération épique » - argument tautologique valant à peu près ce que vaut celui de la « licence poétique », - on peut voir là aussi un souvenir, peut-être lointain, mais surtout une évocation, non moins précise, d'une technique d'embaumement ressemblant étrangement à la momification égyptienne.

Reste à expliquer l'allusion au rouge, qui demeure ambiguë. Peut-être peut-on penser que c'est la couleur du sang et de la vie. Sa présence symbolique est attestée dans bien des cultures, soit sur les corps ou les os, soit dans le décor même de la sépulture ${ }^{18}$. Quant au nitre ou natron, on sait que c'est un sel blanc ou incolore auquel on ne peut imputer la coloration rouge qu'en émettant l'hypothèse d'une contamination par l'oxyde de fer, contenu éventuellement dans les argiles voisines. Les terres du delta pourraient, effectivement, accréditer cette possibilité. Cependant dans l'ignorance où nous sommes de l'origine et du traitement de ce natron, la plus grande prudence s'impose.

Enfin, les relations, au moins sporadiques, entre l'Égypte et la Grèce dès l'époque créto-mycénienne n'étant plus à démontrer, rappelons que Schliemann mentionnait déjà combien le troisième corps de la tombe à fosse $\mathrm{V}$ à Mycènes était merveilleusement préservé et combien sa couleur lui évoquait les momies d'Égypte ${ }^{19}$. D'ailleurs le terme $\tau \alpha \rho \chi v ́ \omega$ utilisé pour désigner les funérailles de Sarpédon, après traitement du corps à l'ambroisie par Apollon, s'il reste vague et continue à faire problème, n'en évoque pas moins le verbe $\tau \alpha \rho \imath \chi \varepsilon u ́ \omega$ qui, dès Hérodote en son livre II, désigne la pratique égyptienne.

16 Notons pourtant qu'à la fin de l'hellénisme antique, dans le corpus médical, le terme d'ambroisie désigne différents onguents, ophtalmiques et autres, rappelant ainsi l'usage le plus ancien des occurrences homériques que l'on a vues. Voir notamment Galien (éd. Kühn, Leipzig, 1824), VIII, p. 292, 1. 4-7, où l'« ambroisie 》 est associée à un produit qu'il faut introduire dans les narines du patient. Le même auteur définit ailleurs (op. cit, Kühn, XI, p. 824, 1. 2-3) l'a $\mu \beta \rho o \sigma i \alpha$ comme un onguent reconnu pour ses effets

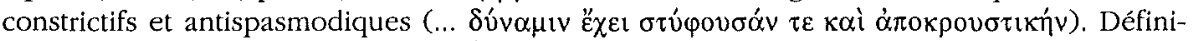
tion reprise à la lettre par Paul d'Égine (Corpus medicorum graeconum, éd. Heilberg, Teubner, 1924, IX, 2, p. 192, 1. 13), = Epitomae medicae, VII, 3 ainsi que par Aétios d'Amida, Iatrorum liber, I, 25 (= TLG 0718. TXT, 1). Une allusion au nectar (sous la forme

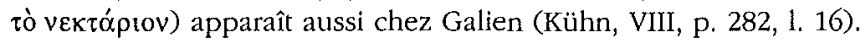

17 HDT., II, 86, 11-13.

18 Voir KuRTZ - BoARdMan, op. cit. (n. 7), p. 216-7.

19 H. Schliemann, Mycènes, Paris, 1879, trad. J. Girardin, p. 377-378. 
Certes, l'analogie n'a pas force de loi, mais en pareilles matières, faut-il vraiment vouloir légiférer ? Cette accointance lexicale, déjà remarquée par $\mathrm{Helbig}^{20}$, peut venir renforcer encore les présomptions que nous voyons doucement se construire.

Mais il est temps de conclure, et de rester sobre. Dans la tradition de la Grèce classique encore, et notamment chez Platon, l'Égypte, non moins que la Crète, a toujours fait figure de haute antiquité et donc de référence à la fois indiscutable et vénérable. On peut penser qu'il en était de même au temps d'Homère et que le Poète a pu, dans le cadre d'une pensée aussi respectueuse de ses racines, en charrier au moins l'un ou l'autre souvenir précis, circonstancié, dûment relayé par cette idéologie mycénienne qui ne cesse de transparaître à fleur d'épopée. Ainsi, les Keftiou de l'époque du bronze ont pu, lors de contacts commerciaux désormais bien établis, s'étonner et s'émerveiller eux aussi, et comme le fera bientôt Hérodote, de certaines techniques mortuaires, qui ne sont en fin de compte que d'étranges et dérisoires essais de nier la mort. Le rêve d'immortalité, vieux comme l'humanité, aura trouvé là matière à réflexions et à fantasmes. D'où le caractère « divin » des interventions thanatologiques que l'on a vues et le recours, constant, qu'elles supposent à ces produits-miracles que sont le nectar et l'ambroisie. Certes, il se peut, malgré la remarque de Schliemann, que les Grecs n'aient jamais momifié leurs morts et que s'ils les ont embaumés, c'était le plus souvent pour préserver le corps le temps d'une protbesis c'est-à-dire un maximum de trentesix à quarante-huit heures. Il n'en reste pas moins que leurs dieux avaient sans doute connu un autre héritage et qu'il leur est arrivé, par exemple dans l'lliade, d'en faire bénéficier quelques mortels d'élection, guerriers exem-

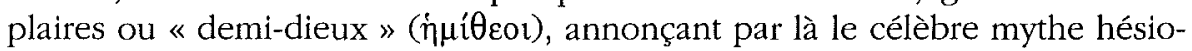
dique de l'« âge des héros».

Pierre SOMVILLE

Université de Liège

Sciences de l'Antiquité

Place du 20-Août, 32

B - 4000 LIÈGE

20 Das Homeriscbe Epos (aus den Denkmälern erläutert), Leipzig, Teubner, 1887, p. 55-56, même s'il ne s'agit pas simplement d'« évidente synonymie » comme le dit l'auteur. 